\title{
T-cell-directed treatment strategies for Type 1 diabetes and the confounding role of inflammation
}

\author{
"Short-term administration of the triple-therapy regimen ... or $\alpha 1$ anti-trypsin ... \\ create resilient drug-free remissions from overt diabetes through ablation of insulitis, \\ restoration of immune tolerance to $\beta$-cells and the unforeseen relief from \\ an inflammatory state in insulin-responsive tissues that, if not corrected, \\ impairs the response of these tissues to insulin."
}

Described by Arataeus as "a melting down of flesh and limbs into urine", the etiology of diabetes long remained a mystery. From the time of Arataeus, approximately 3500 years passed until the disease was controversially linked, at least initially, to malfunction of the pancreas and soon thereafter linked to a failure to produce insulin. The discovery of insulin and the availability of insulin as a therapy changed the fate of millions of patients affected by this condition; however, treatment with exogenous insulin does not provide patients with Type 1 diabetes mellitus (T1DM) protection from long-term morbid complications. In humans and nonobese diabetic (NOD) mice with T1DM, autoimmune $\mathrm{T}$ cells infiltrate and destroy insulin-producing $\beta$-cells, thereby creating an insulin-deficient state [1]. The hypothesis that pancreas transplantation or, even better, transplantation with insulin-producing $\beta$-cells harvested from pancreatic islets represents a better therapeutic solution than exogenous insulin treatment was successfully tested by Paul Lacy [2,3]. Since islets possess both glucose sensors and insulin-secreting cells, tight control of hyperglycemia can often be established with a successful transplant.

The major challenge in islet transplantation remains the prevention of short- and long-term immune- and nonimmune-mediated destruction of the transplanted insulin-producing cells. Moreover, several important immunosuppressive agents have complex diabetogenic properties, including direct $\beta$-cell toxicity and creation of insulin resistance. Despite considerable theoretic advantages for the treatment of T1DM, development of islet transplantation as routine therapy for T1DM has proven difficult. A major step forward was the development of methods for islet purification by Ricordi and colleagues [4]. Through use of the Ricordi method, isolation of $\beta$-cells capable of insulin production post-transplantation was enabled. However, early results were very disappointing, as only few individuals with T1DM were rendered free from exogenous insulin therapy. A major advance was made by the Edmonton group in 2000 [5]. Recognizing that most islets perish in the harvest, purification or as a consequence of nonimmunologic loss in the peritransplant period, the Edmonton group sequentially transplanted islets from multiple donors until the recipient was rendered euglycemic. Hyperglycemia can be controlled such that most patients ( 85-90\%) can discontinue insulin therapy. With time, however, there is an unacceptably high rate of graft failure and $90 \%$ of patients must restart insulin therapy [6]. Compared with whole pancreas transplantation, islet transplantation is bedeviled by the requirement for multiple, not single, donors and inferior long-term outcomes. The barriers to success appear to include: the tremendous nonimmunologic loss of islets during the harvest, culture and immediate post-transplant period that are far more profound than noted with wholepancreas transplantation; and immunologic islet destruction created by rejection and/or recurrence of $\beta$-cell-directed autoimmunity.

\section{"Since islets possess both glucose sensors and insulin-secreting cells, tight control of hyperglycemia can often be established with a successful transplant."}

Restoration of euglycemia, at least temporarily, can be achieved in some new-onset T1DM patients. It is important to note that the advent of overt hyperglycemia occurs before the complete loss of insulin-producing $\beta$-cells. At the

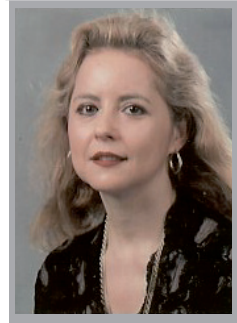

Maria Koulmanda

Author for correspondence: Harvard Medical School, Beth Israel Deaconess Medical Center, Transplant Institute; E/CLS 609, 330 Brookline Ave, Boston, MA, USA Tel.: +16177352882 Fax: +16177352903 mkoulman@bidmc.harvard.edu

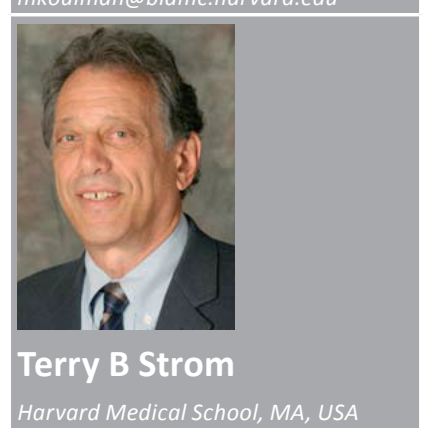


time of onset of frank hyperglycemia, approximately $75-80 \%$ of the $\beta$-cell mass has been destroyed or rendered dysfunctional. For a very short period following onset of hyperglycemia, immunosuppressive therapy, particularly in young patients with T1DM, can produce a remission of the hyperglycemic state. As longterm insulin therapy, particularly through use of highly efficient insulin pumps, is probably safer than long-term treatment with immunosuppressives, the use of long-term maintenance immunosuppression as a means to treat T1DM has been abandoned. The goal of clinical immunologists has turned to efforts to restore immune self-tolerance to $\beta$-cells and foster healing or regeneration of $\beta$-cells. Many immunosuppressives are not suitable for the induction of immune tolerance. Why? Conventional immunosuppressives, including calcineurin inhibitors and corticosteroids, inhibit the expansion and/ or effector function of both tissue-destructive effector and tissue-protective immunoregulatory $\mathrm{T}$ cells. Following the cessation of therapy, the cohort of tissue protective cells has not gained sufficient strength to restrain the $\beta$-cell destructive cells. Hence, the remissions are not sustained for long following withdrawal of the drug because treatment does not produce a state in which islet-reactive regulatory $\mathrm{T}$ cells dominate over islet-reactive cytodestructive immunity and inflammation. However, ablation of the T-celldependent autoimmune disease activity during active treatment does provide islets a respite from the cytodestructive autoimmune process. Recently, attempts to restore tolerance to autologous islets, by tilting the balance of autoimmunity from a tissue-destructive mode toward a tissue-protective mode, have been attempted through short application of monoclonal antibodies rather than small-drug pan-immunosuppressives. Successful immunosuppression is produced through the application of carefully designed humanized anti-CD3. As a consequence, a critical mass of surviving, albeit dysfunctional, islets heal and resume insulin production. In the long term, however, the $\beta$-cell mass does not return to normal and eventually patients must resume insulin therapy [7].

The NOD T1DM model shares many features with human T1DM, including common susceptibility genes and a similar pattern of T-cell-dependent anti- $\beta$-cell immunity $[1,8]$. In the NOD model, the loss of immune tolerance to $\beta$-cells creates vulnerability to autoimmune mediated destruction of insulin-producing $\beta$-cells. Few T-cell-directed therapies have succeeded in restoring euglycemia and selftolerance to islets in overtly diabetic NOD mice [9-12]. We suspect that the inability of many T-cell-directed treatments to quench and control proinflammatory responses, which are not directly mediated by $T$ cells, results in the failure of these T-cell-directed treatments to restore euglycemia and immune tolerance to $\beta$-cells.

\section{"We ... suspect that resolution of detrimental inflammation residing within insulin-sensitive tissues may be helpful in restoring euglycemia in hosts with [Type 1 diabetes mellitus]."}

While many therapeutic interventions can prevent T1DM or resolve the T-cell-rich $\beta$-cellinvasive insulitis lesion in prediabetic hosts, surprisingly few therapies have succeeded in restoring long-term drug-free euglycemia and immune tolerance to $\beta$-cells in overtly diabetic NOD mice. Prior to our studies, only shortterm treatment with anti-CD3 monoclonal antibody [9], polyclonal antilymphocyte serum plus exendin- 4 or infusion of islet-specific regulatory $\mathrm{T}$ cells [12] had proven effective in restoring a long-term euglycemic state in at least $50 \%$ of treated new-onset diabetic NOD mice in the absence of islet transplantation. While both successful therapies directly target $T$ cells, each bears a readily identifiable element that dampens inflammatory responses or their consequences on target tissues. For example, the success of anti-CD3 therapy in the NOD model rests upon its ability to stimulate expression of TGF- $\beta$ by $T$ cells $[9,13]$. It is now well known that the presence of certain inflammatory cytokines within the milieu of antigen recognition by $\mathrm{T}$ cells can direct the commitment of antigen-activated $\mathrm{CD}^{+}{ }^{+} \mathrm{T}$ cells to one of several specific effector or Foxp $3^{+}$regulatory phenotypes [14-16]. In addition, islets are sensitive to proinflammatory cytokines [17-20].

We believe that control of detrimental forms of inflammation, that isinflammation that guides $T$ cells away from a tolerant and toward a cytoprotective format, is crucial to restoring self-tolerance to islets. We also suspect that resolution of detrimental inflammation residing within insulin-sensitive tissues may be helpful in restoring euglycemia in hosts with T1DM. To this end, we have applied triple therapy and $\alpha 1$ anti-trypsin (AAT) in NOD mice with new-onset T1DM. 
Triple therapy is a regimen that powerfully promotes long-term allograft survival/tolerance in daunting transplant models including the model in which allogeneic islets are transplanted into overtly new-onset T1DM NOD hosts in mice [21]. This regimen consists of: an agonist (wild-type) IL-2-Fc fusion protein as a component to enhance activation-induced cell death of effector, but not regulatory, T cells $[22,23]$ and IL-2-mediated and viability differentiation of $\left(\right.$ Foxp $\left.^{+}\right)$regulatory $\mathrm{T}$ cells; a high-affinity IL-15Ra antagonist, mutant IL-15-Fc fusion protein (mIL-15-Fc) [24,25] to block proliferation and promote passive cell death of activated effector T cells $[21,22,26]$ and to block the ability of IL-15 to induce expression of proinflammatory cytokines by activated mononuclear inflammatory cells [23]; and rapamycin to blunt the proliferative response of activated $\mathrm{T}$ cells to T-cell growth factors without inhibiting the activation-induced cell death signal imparted by IL-2 [27] or IL-2-Fc. This triple therapy regimen that selectively targets activated, but not resting, effector $\mathrm{T}$ cells has proven effective in extremely stringent transplant models [23].

Following experience with triple therapy, a regimen known to possess a potent, albeit select, anti-inflammatory element, we directly tested the hypothesis that select anti-inflammatory agents can direct immunity from a cytodestructive to cytoprotective mode and thereby restore self-tolerance and euglycemia. To this end, we treated new-onset overtly diabetic mice with a short course of human AAT. AAT is an acute-phase reactant with antiproteinase [28], anti-inflammatory, antileukocyte migratory and antiapoptotic effects [29-33]. As expression of AAT, a potent anti-inflammatory protein, sharply rises in response to inflammation [34], it seems reasonable to speculate that the function of this protein is to limit the duration, magnitude and perhaps molecular texture of inflammation.

Short-term administration of the tripletherapy regimen [21] or AAT [35] create resilient drug-free remissions from overt diabetes through ablation of insulitis, restoration of immune tolerance to $\beta$-cells and the unforeseen relief from an inflammatory state in insulin-responsive tissues that, if not corrected, impairs the response of these tissues to insulin. A 14- or 28-day course of triple therapy restored an enduring euglycemic state in 55 out of 60 treated, spontaneously and acutely diabetic NOD mice within 5-7 weeks of treatment initiation. In parallel, the autoimmune islet-destructive T-cell-rich insulitis process was aborted and a discriminating state of immune tolerance to 'self'-islet $\beta$-cells was restored. While triple-therapy treatment destroys or inactivates $\beta$-cell-destructive T-cell populations, deletion of the regulatory T-cellrich population of $\mathrm{CD} 25^{+} \mathrm{T}$ cells prior to the triple-therapy treatment precludes restoration of a euglycemic state in triple-therapy treated newonset diabetic NOD mice. The importance of preservation of the regulatory T-cell-rich CD25+ T-cell populations following triple therapy was also evident in the NOD passive transfer model. Overall, the triple-therapy regimen induces specific tolerance and tips the immune balance from diabetogenic toward $\beta$-cell-protective immunity. Restoration of euglycemia may require ablation of an inflammation imposed insulin-resistant state, as well as halting the destructive insulitis and restoration of immune self-tolerance to $\beta$-cells. Triple therapy, a regimen that possesses both immune tolerizing [21] and select anti-inflammatory activities [23], may serve as a prototype for regimens that are well suited for use in restoring euglycemia in individuals with new-onset T1DM.

\section{"...a marked decrease in expression of proinflammatory cytokines is associated with, and perhaps causal for, restoration of immune tolerance to islets."}

$\alpha 1$ anti-trypsin therapy induces tolerance to allogeneic islet transplants [31] and confers antiapoptotic effects upon islets. In association with induction of islet allograft tolerance, Lewis et al. demonstrated that AAT exerts potent islet cytoprotection as well [31]. As we demonstrate herein, human AAT therapy, despite the known immunogenicity of human AAT in mice properties, quickly halts invasive and cytodestructive insulitis-type autoimmunity in the NOD model. Both euglycemia and immune tolerance to $\beta$-cells are restored. The ability of AAT therapy to modify the molecular context in which autoantigen is recognized by $\mathrm{T}$ cells may play an important role in quenching destructive autoimmunity. The cytokine and inflammatory texture of the environment in which naive $\mathrm{CD} 4^{+} \mathrm{T}$ cells recognize antigen dictates the commitment of these cells to various effector (Th1, Th2 and Th17) or Foxp $3^{+}$ regulatory phenotypes [14-16]. Following AAT therapy, an islet-invasive form of insulitis was supplanted by a circumferential type of insulitis that is often associated with tolerance to 
islets $[1,8]$. Indeed, AAT-treated NOD mice are rendered tolerant to syngeneic islets. The rapid ablation of invasive insulitis and the marked decrease in proinflammatory cytokine, but not Foxp3, gene expression within the pancreatic lymph node suggest that AAT-triggered alterations in inflammation can rapidly alter the vigor and fundamental nature of T-celldependent autoimmunity. In short, a marked decrease in expression of proinflammatory cytokines is associated with, and perhaps causal for, restoration of immune tolerance to islets.

\section{"[ $\alpha 1$ anti-trypsin] treatment restores insulin sensitivity and signaling as a consequence of treatment-induced effects upon inflammation."}

Infiltration of activated macrophages or expression of proinflammatory cytokines and proteins within critical insulin-sensitive tissue is known to hamper insulin responsiveness and insulin signaling in obesity-linked T2DM [19,36]. Chaparro et al. demonstrated that new-onset T1DM NOD mice do indeed manifest an insulin-resistant state [37]. Under normal conditions, insulin stimulates disposal of blood glucose into muscle, fat and, to a lesser extent, other insulin-sensitive tissues. A molecular hallmark of insulin-driven glucose disposal is the insulin-triggered tyrosyl phosphorylation of the insulin receptor and immediate downstream signaling molecules within critical insulin-sensitive tissues (e.g., fat and muscle). In obesity-related T2DM [38], a deficiency in insulin-driven glucose disposal is accompanied by, and probably arises as a consequence of, faulty phosphorylation of the insulin receptor. The proximal cause of the insulin resistance and linked faulty tyrosyl phosphorylation of the insulin receptor in obesity-linked T2DM state is subtle inflammation within critical insulin-sensitive tissues. In fact, we note both insulin resistance and gross hypophosphorylation of the insulin receptor in muscle tissues of new-onset T1DM NOD mice. A role for inflammation residing in tissues responsible for insulin-dependent disposal of blood glucose has been linked to a state of faulty insulin signaling and insulin resistance [39] in T2DM [19] and more recently in T1DM [10,37]. Recently, an insulin-resistant state, more classically noted as a feature of T2DM, has been discovered in new-onset T1DM NOD mice $[10,37]$. Both insulin resistance and hypophosphorylation of the insulin receptor were corrected in parallel by AAT treatment. By contrast, restoration of euglycemia with intense insulin treatment did not produce a remission in either insulin resistance or hypophosphorylation of the insulin-activated insulin receptor. These data suggest that the prompt relief from hyperglycemia induced by AAT treatment was linked, at least in part, to resolution of the faulty insulin signaling and insulin resistance. This situation also pertained to our observations with a triple-therapy regimen [10]. Hence, it seems likely that the ability of both AAT and the tripletherapy regimen [10] to relieve faulty insulin signaling and insulin resistance is linked to their ability to restore euglycemia. The presence of both Type 1-like autoimmune $\beta$-cell destruction and Type 2-like insulin resistance may suggest that two separate disease processes converge to create hyperglycemia before the advent of total $\beta$-cell destruction in the NOD model. We now present evidence indicating that an AAT-sensitive inflammatory state is the probable proximal cause of both autoimmunity and insulin resistance. Hence, we propose that a proinflammatory state present in NOD mice triggers both autoimmunity and insulin resistance.

While triple-therapy treatment ablated aggressive insulitis, the restoration of euglycemia in power-mix-treated NOD mice was not associated with a dramatic increase in the $\beta$-cell mass. Hence, we probed for other mechanisms that may contribute to restoration of euglycemia. We tested the hypothesis that insulin resistance present in new-onset T1DM NOD mice may be linked by expression of proinflammatory molecules within fat and muscle, which are crucial for insulin-triggered disposal of glucose, and that resolution of an inflammationassociated insulin-resistant state and of faulty insulin-triggered tyrosine phosphorylation of insulin-signaling molecules may be linked to restoration of euglycemia. For these reasons, we have tested the hypothesis that treatment with triple therapy or AAT, an acute-phase reactant with potent anti-inflammatory, antileukocyte migratory and anti-apoptotic effects [29-32] including effects upon islets [31,33], is efficacious for NOD mice with overt new-onset T1DM. In short, we were probing the hypothesis that inflammatory mechanisms trigger the loss of islet tolerance, invasive insulitis, insulin resistance and faulty insulin signaling in T1DM. 
Note that the functional, insulin-positive $\beta$-cell mass expanded markedly and rapidly in AAT-treated hosts. These findings tend to further authenticate the potent AAT-fostered cytoprotective effects upon islets as demonstrated by Lewis et al. [31]. Indeed, this is the first demonstration in which successful treatment of new-onset diabetic mice in the absence of islet-cell transplantation routinely leads to expansion of the $\beta$-cell mass.

The interactive network-based functional analysis of DNA microarray data of fat tissue obtained from normal, diabetic and AAT-treated mice provides an insight into the association between the effects of AAT treatment and the impact of treatment upon biological processes related to nucleic acid/lipid metabolism, immune response and inflammatory disease. The data strongly suggest that AAT treatment restores insulin sensitivity and signaling as a consequence of treatment-induced effects upon inflammation. Nevertheless, none of these genes (TNF- $\alpha, I L-4$ and $N F-\kappa \mathrm{B})$ were identified as differentially expressed on Affymetrix arrays. Reverse-transcription PCR, a more sensitive technique, did detect a suppressive effect of AAT therapy upon intra-fat $T N F-\alpha$, but not $I L-4$, gene expression. As the regulation of NF- $\kappa \mathrm{B}$ activation is post-transcriptional, we did not assess the effect of AAT treatment on gene expression.

\section{Financial \& competing interests disclosure}

Maria Koulmanda and Terry B Strom have received grant support from the NIH (NIPH PPG U19 DK080652; NIH P01 AI041521) and the Juvenile Diabetes Research Foundation (JDRF 4-2004-1057; JDRF 7-2005-1329). The authors have no other relevant affiliations or financial involvement with any organization or entity with a financial interest in or financial conflict with the subject matter or materials discussed in the manuscript apart from those disclosed. No writing assistance was utilized in the production of this manuscript.

\section{Bibliography}

1 Rossini AA, Mordes JP, Like AA: Immunology of insulin-dependent diabetes mellitus. Annu. Rev. Immunol. 3, 289-320 (1985).

2 Ballinger WF, Lacy PE: Transplantation of intact pancreatic islets in rats. Surgery 72 , 175-186 (1972).

3 Lacy PE, Kostianovsky M: Method for the isolation of intact islets of Langerhans from the rat pancreas. Diabetes 16, 35-39 (1967).

4 Ricordi C, Strom TB: Clinical islet transplantation: advances and immunological challenges. Nat. Rev. Immunol. 4, 259-268 (2004).

5 Shapiro AM, Lakey JR, Ryan EA et al.: Islet transplantation in seven patients with Type 1 diabetes mellitus using a glucocorticoid-free immunosuppressive regimen. N. Engl. J. Med. 343, 230-238 (2000).

6 Shapiro AM, Ricordi C, Hering BJ et al.: International trial of the Edmonton protocol for islet transplantation. N. Engl. J. Med. 355, 1318-1330 (2006).

7 Herold KC, Hagopian W, Auger JA et al.: Anti-CD3 monoclonal antibody in new-onset Type 1 diabetes mellitus. N. Engl. J. Med. 346, 1692-1698 (2002).

8 Shoda LK, Young DL, Ramanujan S et al.: A comprehensive review of interventions in the NOD mouse and implications for translation. Immunity 23, 115-126 (2005).

9 Belghith M, Bluestone JA, Barriot S et al: TGF- $\beta$-dependent mechanisms mediate restoration of self-tolerance induced by antibodies to CD3 in overt autoimmune diabetes. Nat. Med. 9, 1202-1208 (2003).
10 Koulmanda M, Budo E, Bonner-Weir S et al.: Modification of adverse inflammation is required to cure new-onset Type 1 diabetic hosts. Proc. Natl Acad. Sci. USA 104, 13074-13079 (2007).

11 Ogawa N, List JF, Habener JF, Maki T: Cure of overt diabetes in NOD mice by transient treatment with anti-lymphocyte serum and exendin-4. Diabetes 53, 1700-1705 (2004).

12 Tarbell KV, Petit L, Zuo X et al.: Dendritic cell-expanded, islet-specific $\mathrm{CD} 4{ }^{+} \mathrm{CD} 25^{+}$ $\mathrm{CD}^{2} \mathrm{~L}^{+}$regulatory $\mathrm{T}$ cells restore normoglycemia in diabetic NOD mice. J. Exp. Med. 204, 191-201 (2007).

13 Bresson D, Togher L, Rodrigo E et al.: Anti-CD3 and nasal proinsulin combination therapy enhances remission from recent-onset autoimmune diabetes by inducing Tregs. J. Clin. Invest. 116, 1371-1381 (2006).

14 Bettelli E, Carrier Y, Gao W et al.: Reciprocal developmental pathways for the generation of pathogenic effector TH17 and regulatory T cells. Nature 441, 235-238 (2006).

15 Tato CM, O'Shea JJ: Immunology: what does it mean to be just 17? Nature 441, 166-168 (2006).

16 Veldhoen M, Hocking RJ, Atkins CJ, Locksley RM, Stockinger B: TGF $\beta$ in the context of an inflammatory cytokine milieu supports de novo differentiation of IL-17producing T cells. Immunity 24, 179-189 (2006).

17 Barshes NR, Wyllie S, Goss JA: Inflammation-mediated dysfunction and apoptosis in pancreatic islet transplantation: implications for intrahepatic grafts. J. Leukoc. Biol. 77, 587-597 (2005).
18 Eizirik DL, Mandrup-Poulsen T: A choice of death - the signal-transduction of immune-mediated $\beta$-cell apoptosis. Diabetologia 44, 2115-2133 (2001).

19 Hotamisligil GS: Inflammation and metabolic disorders. Nature 444, 860-867 (2006).

20 Sandler S, Andersson A, Hellerstrom C: Inhibitory effects of interleukin 1 on insulin secretion, insulin biosynthesis, and oxidative metabolism of isolated rat pancreatic islets. Endocrinology 121, 1424-1431 (1987).

21 Zheng XX, Sanchez-Fueyo A, Sho M et al: Favorably tipping the balance between cytopathic and regulatory $\mathrm{T}$ cells to create transplantation tolerance. Immunity 19 , 503-514 (2003).

22 Fontenot JD, Rasmussen JP, Gavin MA, Rudensky AY: A function for interleukin 2 in Foxp3-expressing regulatory $\mathrm{T}$ cells. Nat. Immunol. 6, 1142-1151 (2005).

23 Zheng XX, Maslinski W, Ferrari-Lacraz S, Strom TB: Cytokines in the treatment and prevention of autoimmune responses - a role of IL-15. Adv. Exp. Med. Biol. 520, 87-95 (2003).

24 Ferrari-Lacraz S, Zheng XX, Kim YS et al: : An antagonist IL-15/Fc protein prevents costimulation blockade-resistant rejection. J. Immunol. 167, 3478-3485 (2001).

25 Waldmann TA, Dubois S, Tagaya Y: Contrasting roles of IL-2 and IL-15 in the life and death of lymphocytes: implications for immunotherapy. Immunity 14, 105-110 (2001). 
26 Li Y, Li XC, Zheng XX et al.: Blocking both signal 1 and signal 2 of T-cell activation prevents apoptosis of alloreactive $\mathrm{T}$ cells and induction of peripheral allograft tolerance. Nat. Med. 5, 1298-1302 (1999).

27 Zheng XX, Steele AW, Hancock WW et al.: IL-2 receptor-targeted cytolytic IL-2/Fc fusion protein treatment blocks diabetogenic autoimmunity in nonobese diabetic mice. J. Immunol. 163, 4041-4048 (1999).

28 Breit SN, Wakefield D, Robinson JP et al: The role of $\alpha 1$-antitrypsin deficiency in the pathogenesis of immune disorders. Clin. Immunol. Immunopathol. 35, 363-380 (1985).

29 Churg A, Dai J, Zay K et al.: $\alpha$-1-antitrypsin and a broad spectrum metalloprotease inhibitor, RS113456, have similar acute anti-inflammatory effects. Lab. Invest. 81, 1119-1131 (2001).

30 Jie Z, Cai Y, Yang W et al.: Protective effects of $\alpha 1$-antitrypsin on acute lung injury in rabbits induced by endotoxin.

Chin. Med. J. (Engl.) 116, 1678-1682 (2003).
31 Lewis EC, Shapiro L, Bowers OJ, Dinarello CA: $\alpha 1$-antitrypsin monotherapy prolongs islet allograft survival in mice. Proc. Natl Acad. Sci. USA 102, 12153-12158 (2005).

32 Petrache I, Fijalkowska I, Zhen L et al.: A novel antiapoptotic role for $\alpha 1$-antitrypsin in the prevention of pulmonary emphysema. Am. J. Respir. Crit. Care Med. 173, 1222-1228 (2006).

33 Abbas AK, Corson JM, Carpenter CB et al.: Immunologic enhancement of rat renal allografts. III. Immunopathologic lesions and rejection in long-surviving passively enhanced grafts. Am. J. Pathol. 79, 255-270 (1975).

34 Brantly M: $\alpha 1$-antitrypsin: not just an antiprotease: extending the half-life of a natural anti-inflammatory molecule by conjugation with polyethylene glycol. Am. J. Respir. Cell Mol. Biol. 27, 652-654 (2002).
35 Koulmanda M, Bhasin M, Hoffman L et al.: Curative and $\beta$ cell regenerative effects of $\alpha 1$-antitrypsin treatment in autoimmune diabetic NOD mice. Proc. Natl Acad. Sci. USA 105, 16242-16247 (2008).

36 Bruning JC, Winnay J, Bonner-Weir $\mathrm{S}$ et al.: Development of a novel polygenic model of NIDDM in mice heterozygous for IR and IRS-1 null alleles. Cell 88, 561-572 (1997).

37 Chaparro RJ, Konigshofer Y, Beilhack GF et al:: Nonobese diabetic mice express aspects of both Type 1 and Type 2 diabetes. Proc. Natl Acad. Sci. USA 103, 12475-12480 (2006).

38 Shoelson SE, Lee J, Goldfine AB: Inflammation and insulin resistance. J. Clin. Invest. 116, 1793-1801 (2006).

39 Hotamisligil GS, Shargill NS, Spiegelman BM: Adipose expression of tumor necrosis factor- $\alpha$ : direct role in obesity-linked insulin resistance. Science 259, 87-91 (1993). 